\title{
A technique for anodally blocking large nerve fibres through chronically implanted electrodes
}

\author{
G S B R I N D L E Y A N D M D C R A G G S \\ From the MRC Neurological Prostheses Unit, Institute of Psychiatry, London
}

SUMMARY If a spinal root of a baboon or rhesus monkey is trapped in an initially loosefitting silicone rubber channel containing two or more platinum electrodes, electrical pulses sent through these electrodes can stimulate nerve fibres close to the cathode and block the resulting impulses close to the anode. We show (1) how anodal break excitation and excitation of fibres outside the silicone rubber channel can be avoided; (2) that an implant 16 months old behaves like a recent one; (3) that in a root containing somatic motor fibres and parasympathetic fibres, all somatic motor fibres can be blocked and most or all parasympathetic fibres excited but not blocked; (4) that provided that the electrodes pass no net direct current, prolonged stimulation with block can be harmless; (5) how block can be achieved in one direction only along a root; (6) that a peripheral nerve can be blocked by the same techniques.

It has long been known ${ }^{1}$ that when steady direct current is passed through a nerve, excitability is raised near the cathode and lowered near the anode. From this and related observations arose the belief in the nineteenth century, and later the certain knowledge, ${ }^{2}$ that when a brief pulse of current is passed through a nerve, impulses arise (if at all) near the cathode and are blocked (if at all) near the anode. The threshold for anodal block is always higher than that for excitation. Both these thresholds, for external electrodes, are approximately inversely proportional to the lengthconstant of the fibre investigated, and this (for a myelinated fibre) is approximately proportional to its diameter. ${ }^{3}$ Kuffler and Vaughan Williams ${ }^{4}$ used anodal block to achieve stimulation of the large fibres of a freshly exposed spinal root in one direction only, but of the small fibres in both directions. Their technique, which was to pass rectangular pulses of current between two electrodes, was successful with freshly exposed spinal roots, buit did not work with peripheral nerves because large fibres were excited near the anode when each pulse ceased. Such "anode break excitation" is another long-known phenomenon, clearly referred to by Gotch and Macdonald ${ }^{5}$ in 1896 on p 257 line 9 of a long paper mainly on

Address for reprint requests: Dr GS Brindley, Institute of Psychiatry, de Crespigny Park, London SE5 8AF.

Accepted 30 June 1980 other matters. It was certainly known before Gotch and Macdonald, but we cannot find who discovered it; probably not Pflüger, though he made closely related observations. ${ }^{1}$ Anode break excitation is in part due to the passive electrical properties of the connective tissue sheath, and hence occurs more readily in peripheral nerves than in freshly exposed normal spinal roots, which have no sheath. Extension of the techniques of intermittent anodal block to peripheral nerves was first achieved by Burke and Ginsborg, ${ }^{6}$ who avoided anode break excitation by making each pulse cease gradually instead of suddenly. Burke and Ginsborg used exponential decay with a timeconstant of about $30 \mathrm{~ms}$, but it is possible to use much faster decay; Accornero et al ${ }^{7}$ succeeded in avoiding anode break excitation with pulses whose decay time-constants were $8 \mathrm{~ms}$ (their fig 3) and about $4 \mathrm{~ms}$ (their fig 5B), and we shall show that even shorter time-constants can be used under our conditions.

We have seen no report, except our preliminary communication, ${ }^{8}$ of anodal block achieved with implanted electrodes. Such block could be useful in neurological prosthetic devices. For example, stimulation of sacral anterior roots through implanted electrodes gives excellent emptying of the urinary bladder in anaesthetised baboons and in baboons that have been paraplegic for only a few days or weeks. ${ }^{9}$ But in the application of such a 
technique to human paraplegic patients there may be a difficulty that does not arise in the anaesthetised or newly paraplegic baboon: many such patients have strong tonic activity of the striated muscles that close the urethra, and this activity is not inhibited during detrusor contraction (detrusor-sphincter dyssynergia ${ }^{10}$ ). If sacral anterior roots could be stimulated in such a way that the small parasympathetic fibres fired orthodromically and antidromically, but the larger somatic motor fibres antidromically only, the unwanted largefibre activity could at least be blocked by collision, and if the human sacral cord contains antidromic inhibitory mechanisms like those of the cat's lumbar $\operatorname{cord}^{11} 12$ it could be inhibited. Collision block would require a high frequency of antidromic firing of large fibres, but for antidromic inhibition a much lower frequency should suffice.

In the present paper we identify and solve the special problems that arise in attempting to achieve anodal block in roots of the cauda equina with implanted electrodes. We show how block can be achieved on both sides of the stimulating cathode or on one side only, and establish that the range of pulse amplitudes with which total anodal block can be obtained is large. We can obtain it with pulses large enough to cause a maximal rise in bladder pressure. The roots are not detectably damaged by nearly two hours of application of 50 anodally blocking pulses per second, or $\frac{1}{2}$ hour of 300 per second.

\section{Methods}

Electrodes were implanted on spinal roots in eight baboons and four rhesus monkeys, and on a peripheral nerve in one baboon (see table). The surgical techniques were as described by one of us, ${ }^{9}$ and the electrode-mounts have a general similarity to those there described, but differ in their details. These details for the 11th and 12th implants of table (R15 and R16) are shown in fig 1 . The other implants differ from fig 1 in their electrode spacing (given in table) and in the number of channels (one in the first five implants, four in the next seven, and two in the last).

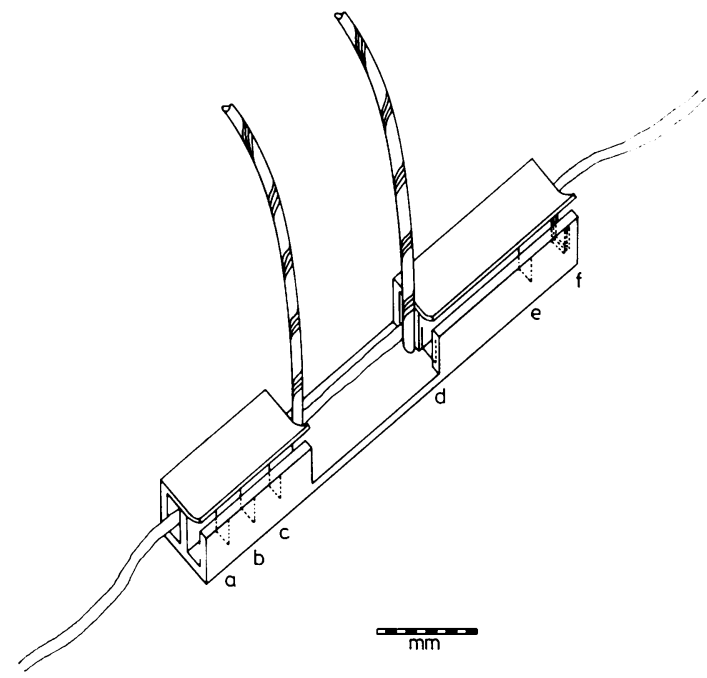

Fig 1 Implanted electrodes: each implanted electrode-mount consists of a silicone-rubber body with two pairs of parallel channels closed at the top by means of silicone rubber flaps. Each channel of the caudal pair containing three asymmetrically placed platinum electrodes $(a-b-c)$, and each channel of the caudal pair containing three asymmetrically placed electrodes $(d-e-f)$. The outer electrodes of each set of three are usually connected together as a common anode. Contact with the electrodes is made through helically wound cables. The nearer channel is ready to receive a nerve whilst the other is shown occupied.

Table Details of experiment

\begin{tabular}{|c|c|c|c|c|c|}
\hline Animal & Electrodes & $\begin{array}{l}\text { Distances between } \\
\text { electrodes }(\mathrm{mm})\end{array}$ & Roots trapped & $\begin{array}{l}\text { Dates of } \\
\text { implantation }\end{array}$ & Dates of experiments \\
\hline $\begin{array}{l}\text { B95F } \\
\text { B91M } \\
\text { B94M } \\
\text { B93F } \\
\text { R12F } \\
\text { B96M } \\
\text { R14M } \\
\text { B101M } \\
\text { B98M } \\
\text { B102F } \\
\text { R15M } \\
\text { R16M } \\
\text { B104M }\end{array}$ & $\begin{array}{l}6 \\
2 \\
4 \\
4 \\
8 \\
5 \\
5 \\
5 \\
6 \\
6 \\
6 \\
6 \\
6\end{array}$ & $\begin{array}{l}4,10,4,17,3 \\
4.5 \\
5,6,3 \\
5,6,3 \\
3.5,2.5,2,2,3.5,4,3 \\
6,27,4,4 \\
6,25,4,4 \\
5,29,4,4 \\
4.5,4.5,35,5,5 \\
4.5,4.5,29,5,5 \\
3.5,3.5,20,3.5,9 \\
4.5,4.5,20,3,12 \\
5,5,19,3.5,10\end{array}$ & $\begin{array}{l}\text { Left L7D } \\
\text { Both S1V } \\
\text { Both S12DV } \\
\text { Both S12DV } \\
\text { Both S12DV } \\
\text { Both S12DV } \\
\text { Both caudal DV } \\
\text { Both S12V } \\
\text { Both S12V } \\
\text { Both S12V } \\
\text { Both S12V } \\
\text { Both S12V } \\
\text { Lateral popliteal } \\
\text { nerve }\end{array}$ & $\begin{array}{l}21.9 .77 \\
24.2 .78 \\
13.4 .78 \\
10.5 .78 \\
24.5 .78 \\
28.6 .78 \\
31.8 .78 \\
30.10 .78 \\
29.11 .78 \\
13.12 .78 \\
19.2 .79 \\
16.3 .79 \\
29.8 .79\end{array}$ & $\begin{array}{l}3.2 .78 \\
10.3 .78,10.4 .78 \\
2.5 .78,9.10 .78,4.7 .79 \\
12.6 .78,20.10 .78,5.7 .79 \\
9.6 .78,23.10 .78,26.1 .79 \\
16.10 .78,12.1 .79 \\
27.10 .78 \\
22.11 .78,16.11 .79,3.3 .80 \\
2.2 .79,21.11 .79 \\
7.9 .79,6.2 .80 \\
30.3 .79,16.7 .79,21.1 .80 \\
30.4 .79 \\
23.11 .79\end{array}$ \\
\hline
\end{tabular}




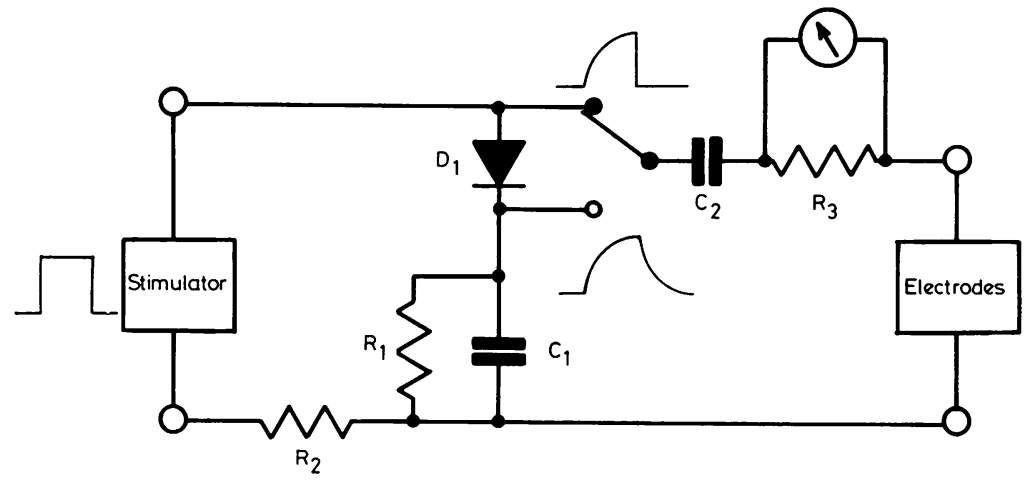

Fig 2 Pulse shaping circuit: the circuit is supplied with rectangular pulses from a conventional electrophysiological stimulator (Two Devices Type 3072 in series or one Devices Mark IV). Each pulse can be made to begin exponentially and end either suddenly or exponentially. For exponential changes the timeconstant of rise is approximately $R_{2} C_{1}$ and that of fall is approximately $R_{3} C_{1} . R_{3}$, across which pulse current is measured, reduces the effect of the electrode capacitance on the shape of the pulse. $C_{2}$, is a large capacitor to block the direct current component of stimulation which would otherwise be harmful to the nerves. $R_{1}$, removes any residual charge on $C_{1}$ between pulses.

$C_{1}: 0 \cdot 1 \mu F$ or other values.

$\mathrm{C}_{2}: 4.7 \mu F$.

$D_{1}: 15 \mathrm{~J} 150$.

$R_{1}: 1 M \Omega$.

$R_{2}: 560 \Omega$ or other values.

$R_{3}: 6.8 \mathrm{~K} \Omega$ or more.

Experiments were done under pentobarbitone anaesthesia from three weeks to 16 months after implantation of electrodes. The only surgical interference needed for an experiment was exposure of the subcutaneous cables of the implant. Each cable was brought out through a skin incision about $5 \mathrm{~mm}$ in length. At the end of an experiment the cables were usually replaced under the skin and the animal allowed to recover from the anaesthetic so that it could be tested again some months later.

Figure 2 shows the circuit by which pulses beginning and ending exponentially were produced. The time constants of beginning and of ending can be adjusted independently.

\section{Results}

Two electrodes

Our early experiments were done with two electrodes, and fig 3 illustrates that anodal block can be obtained with such a system. However, when two electrodes are used, current flows between them not only along the short direct path within the silicone rubber channel that contains them and the root, but also in long looping paths externally. This external current demonstrably stimulates neighbouring roots, which is very disadvantageous in a therapeutic implant. It is theoretically probable that it can also stimulate, beyond the region of anodal block, the root on which the electrodes lie, and thereby cause an apparent breakdown of anodal block at high stimulus strengths.

A tripolar electrode system (cathode between two anodes) should, if perfectly symmetrical, eliminate the external current flow. Experimentally we find that such tripoles greatly reduce unintended stimulation of other roots, and substan-

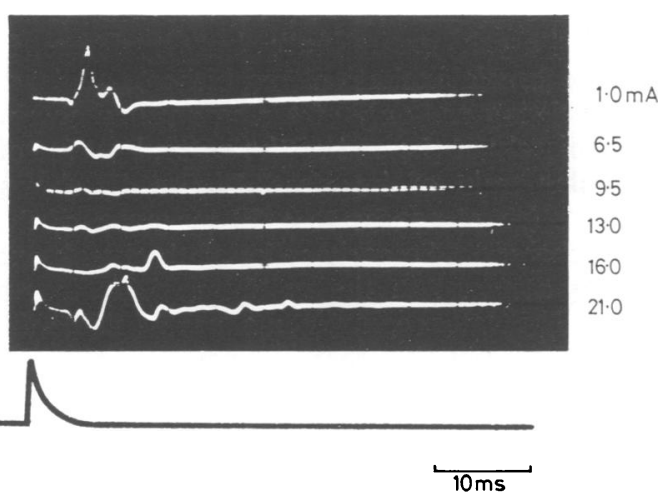

Fig 3 Anodal block with two electrodes: an EMG from a proximal tail muscle, elicited by sacral ventral root stimulation through two electrodes, can be partially blocked, but only over a small range of current strengths. The anode is placed distal to the cathode, that is, nearer to the muscle. The current pulse is shown beneath the oscilloscope records. For these and other non-rectangular pulses the numbers beside the traces are peak currents. Baboon B102, fourteen months after implantation. 
tially increase the range of stimulus strengths at which manifest anodal block occurs. Since dipoles have no advantage over tripoles to compensate for these two disadvantages, we need say no more about them.

Three electrodes, symmetrically placed

In the last eight of the 13 animals of the table the roots or nerve pass through two silicone rubber channels (as shown in fig 1), and in one of these channels (B96, R14, B101, R15, R16, B104) or both of them (B98, B102) there are three electrodes placed symmetrically. The experiments to be described next used such tripoles, the outer electrodes being anodes and the central one cathode.

Figure 4A shows electromyograms (representing many motor units) recorded with wire electrodes in muscle of the proximal third of the tail. Rectangular current pulses lasting $20 \mathrm{~ms}$ were passed through the tripole. At 0.3 and $0.5 \mathrm{~mA}$ peak current there is a nearly maximal spike corresponding to the beginning of the pulse, and probably repetitive firing of some units during later parts of the pulse. At 0.7 to $3.8 \mathrm{~mA}$ the repetitive firing late in the pulse is obvious. At 4.6 $\mathrm{mA}$ the repetitive firing disappears and the early spike is diminshed. This is the beginning of anodal block. At 5.5 to $9 \mathrm{~mA}$ the anodal block during the pulse becomes progressively more complete, but firing occurs after the pulse has finished. The excitation responsible for this late firing may be called "break excitation"; but it differs from "anode break excitation" as hitherto understood in consisting not only of a single volley occurring immediately at the end of the pulse, but also of an irregular train of impulses. Figure 4B shows 15 consecutive responses to a $20 \mathrm{~ms}$ pulse of $9 \mathrm{~mA}$ peak current. The early spike of the break excitation is constant in timing but variable in amplitude. The later components are variable in all respects except that they are never wholly absent. Figure 4B illustrates that they last at least $20 \mathrm{~ms}$. In other experiments we have seen them last up to $80 \mathrm{~ms}$. They represent a long-lasting after-effect either of excessive depolarisation in the cathodal region or of excessive hyperpolarisation in the two anodal regions. We have no evidence that determines whether the depolarisation or the hyperpolarisation is responsible, and both may be involved. The alternative hypothesis that the late impulses arise in the spinal cord is excluded by the experiment of fig 5: they are unaffected if the roots are cut through between the stimulating electrodes and the spinal cord.

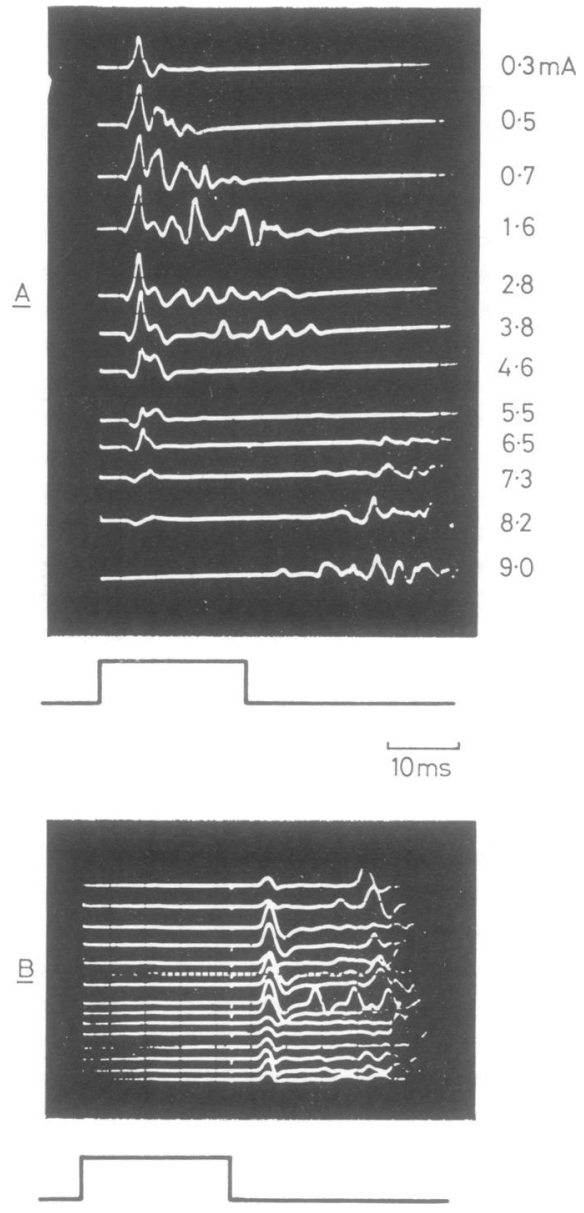

Fig 4 Anodal block uing rectangular current pulses with symmetrically placed electrodes: the outer electrodes of the tripole are connected together as a common anode. $(A)$ Gradually increasing the pulse current blocks the EMG response during stimulation. At higher currents break exc:tation occurs after the pulse has finished. (B) Fifteen consecutive responses at the same optimal current for anodal block. Break excitation is always present. No stimulation of adjacent roots occurs even at high pulse-currents. Baboon B102, fourteen months after implantation.

If a $20 \mathrm{~ms}$ pulse is caused to begin exponentially with a time constant of $0.2 \mathrm{~ms}$ instead of suddenly, the initial anodal block becomes a little less good, but this impairment can be overcome by a slight increase of amplitude. The manner in which the pulse begins has no other notable effects. Causing the pulse to end exponentially with a time-constant of 0.2 to $2 \mathrm{~ms}$ usually abolishes early break ex- 

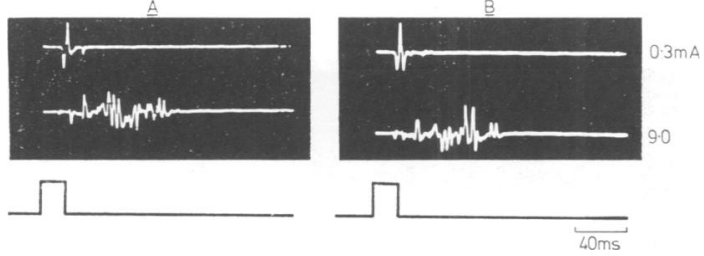

Fig 5 Proof that late break excitation is not reflex: late break excitation is seen to follow anodal block both before $(A)$, and after $(B)$ all spinal roots are cut through between the stimulating electrodes and the spinal cord. EMG from a small muscle of the foot. Baboon B101, sixteen months after implantation.

citation, but does not diminish the late break excitation.

Our failure to eliminate late break excitation with 20 ms pulses excludes the use of such pulses in a bladder-controlling implant. We have other good reasons for choosing much shorter pulses if possible: to reduce power consumption, and to lessen the risk of damaging the nerve fibres.

Figure 6A illustrates that suddenly-ending pulses of amplitude $10 \mathrm{~mA}$ give no block if they last $0 \cdot 1$

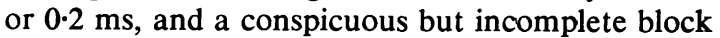
if they last $0.5,1.0$ or $5.0 \mathrm{~ms}$. Experiments like that of fig 6B show that this finding is general for the best-chosen amplitude: at $0.5 \mathrm{~ms}$ or longer durations an amplitude can always be found that gives partial block, but at $\mathbf{0 . 2} \mathrm{ms}$ or shorter durations no such amplitude can be found.

The degree of block can be much improved, and the range of amplitudes over which it occurs can be increased, by causing the pulses to end exponentially instead of suddenly. Figure 7 shows that pulses whose rising phase occupies $0.2 \mathrm{~ms}$, and whose falling phase is exponential with timeconstant $0.9 \mathrm{~ms}$, give complete block over a wide range of amplitudes, 10.0 to $29.0 \mathrm{~mA}$. At still higher amplitudes (last three traces), break excitation, including late break excitation, occurs.

Three electrodes unsymmetrically placed: one-way stimulation

To avoid external current flow, with its harmful effects of stimulating unintended roots and unintended parts of the intended root, it is desirable that the two ends of the silicone rubber channel that contains the root and the electrodes be at the same potential at all times. In a perfectly symmetrical electrode array this is achieved automatically. In an asymmetrical array like d-e-f of fig 1 it will be nearly achieved if at all times the ratio of currents through the two anodes is the
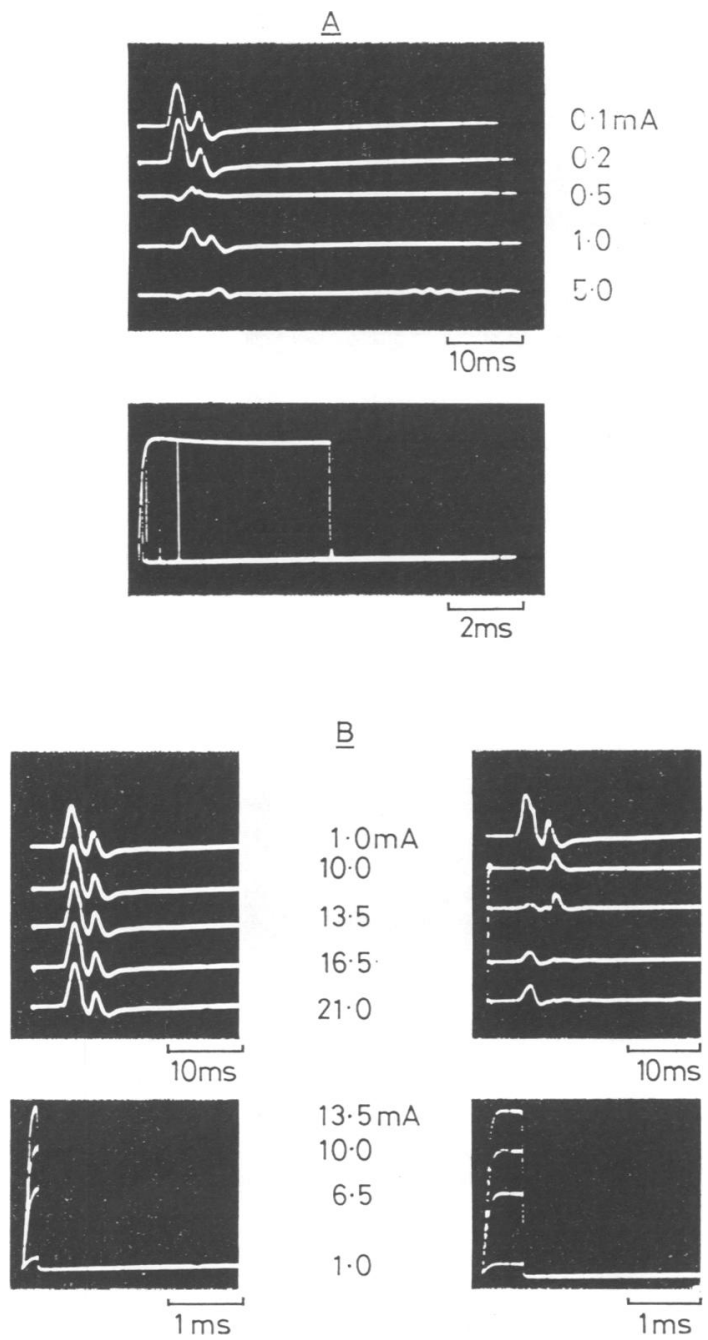

Fig 6 Effect of pulse duration and size on blocking: effect of duration $(A)$ and amplitude $(B)$ on blocking with pulses that end suddenly. In $A$ the amplitude is $10 \mathrm{~mA}$ at the peak (which the two shortest pulses never quite reach). In $B$ the durations are 0.2 and 0.55 (nominally 0.5 ) $\mathrm{ms}$. Baboon B102, fourteen months after implantation.

reciprocal of the ratio of their distances from the cathode. Then for longitudinal current the smaller current in the long limb d-e of the tripole compensates for its greater longitudinal resistance so that the voltage developed is the same as for the short limb f-e. To achieve the right ratio of currents the anode that is nearer to the cathode needs to have a greater area of exposed platinum. By how much it should be greater we determined 

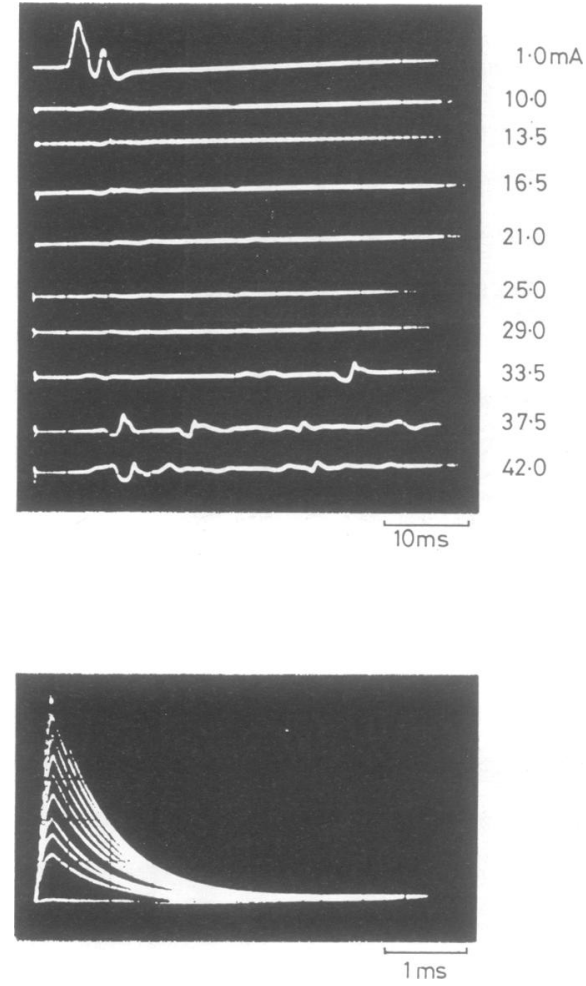

Fig 7 Anodal block with short exponentially-ending pulses: complete block is achieved over a wide range of amplitudes by exponentially-ending current pulses of time-constant $0.9 \mathrm{~ms}$ applied through a symmetrical tripole (outer electrodes connected together as a common anode). Baboon B102, fourteen months after implantation.

roughly by trial; in practice the external balancing of the unsymmetrical array does not need to be very accurate.

An internally asymmetrical array, made by these means to be roughly balanced as viewed externally, always in our experience gives better anodal block under that anode which carries the greater current. Figure 8 illustrates this; the distal anode $f$ carries the greater current, chiefly because of its greater area of wire. Correspondingly the distally conducted volley (shown by the foot electromyogram that it produces) is completely blocked at $5.5 \mathrm{~mA}$, but the centrally conducted volley (shown as electroneurogram from electrodes abc) is unaffected at $5.5 \mathrm{~mA}$ and only partly blocked at $8.5 \mathrm{~mA}$. At $5.5 \mathrm{~mA}$ and slightly higher currents we are sending impulses antidromically but not orthodromically along the $\alpha$ motor fibres.

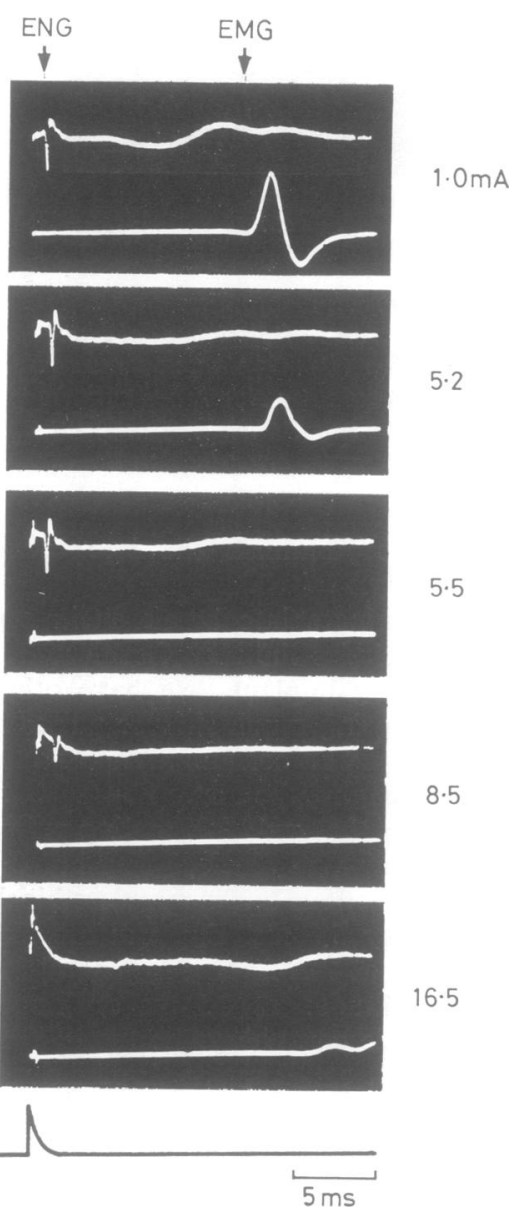

Fig 8 One-way stimulation of spinal roots by means of three electrodes placed asymmetrically: the electroneurogram (ENG) (upper trace) is recorded from the symmetrically placed electrodes $(a-b-c$ of fig 1) closer to the spinal cord. The evoked EMG from a small muscle of the foot (lower trace) is suppressed at $5.5 \mathrm{~mA}$; the ENG is unaffected at this current, and persists at about half amplitude at 8.5 mA. Rhesus monkey R15, eleven months after implantation.

Other experiments (like that of fig 9) show that such stimulation produces orthodromic impulses in preganglionic parasympathetic fibres. Presumably these parasympathetic fibres also fire antidromically, but testing this presumption need not concern us.

In a planned asymmetrical tripole we avoid, for theoretical reasons, interelectrode distances less than the length-constant of the fibres. This length-constant, as estimated from the relation 


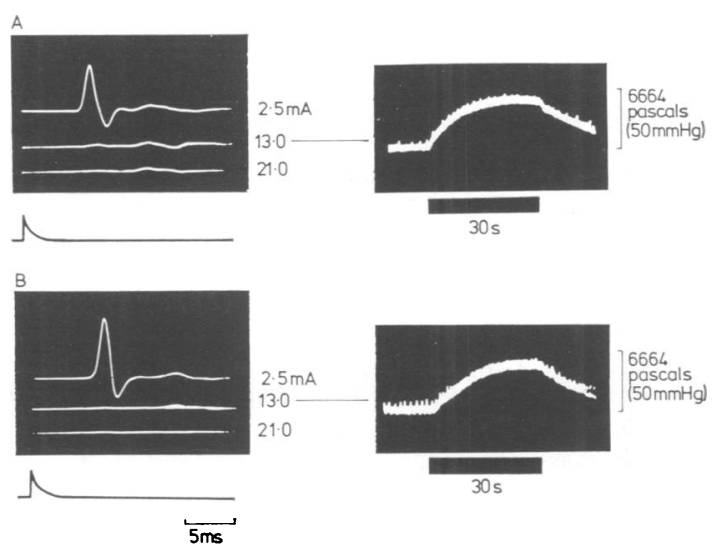

Fig 9 Harmlessness of prolonged intermittent anodal block. (A) Blockade of the large somatic motor fibres occurs with stimulation at 50 exponentially decaying pulses per second, whereas at the same time the smaller parasympathetic motor fibres are not blocked and give a good rise in bladder pressure (bar under bladder pressure is the period of stimulation at 50/s). The toe muscle responses are to single pulses only. (B) As for A but recorded 3 mins (for emg) and 7 mins (for bladder) after 75 min of prolonged intermittent anodal block. Baboon B93, five months after implantation.

between threshold and interelectrode distance in the trapped roots of $R 12$, was $2.2 \mathrm{~mm}$.

After our asymmetrical tripoles had been designed, implanted and tested we learned of the use, in acute experiments, of a very similar arrangement by van den Honert and Mortimer. ${ }^{13}$

\section{The harmlessness of prolonged intermittent} anodal block

If a nerve or root is subjected to prolonged continuous anodal block it must surely suffer electrolytic damage, or at least damage from products of electrolysis diffusing from the electrode surfaces. It seems, however, that the cycles of block and non-block that we employ are relatively harmless. In B93, R12 and R14 we applied, for over an hour, 50 pulses per second of the time-course shown in fig 7 and of ampltiude sufficient to produce complete anodal block of the somatic motor fibres and excitation without block of the parasympathetic fibres to the bladder. There was a $4.7 \mu \mathrm{F}$ capacitor in series with the electrodes $\left(\mathrm{C}_{2}\right.$ of fig 2) to make the long-term average of current zero. The exact times of stimulation were 75 minutes for B93, 95 minutes for $\mathrm{R} 12$, and 115 minutes for R14. After these prolonged assaults, the responses of the motor nerve fibres were severely depressed for the first 10 to 30 seconds, but had completely recovered in 15 minutes or less in all three animals. Figure 9 illustrates this for B93. A similar result was obtained in B91 with 300 anodally blocking pulses per second, applied for 34 minutes.

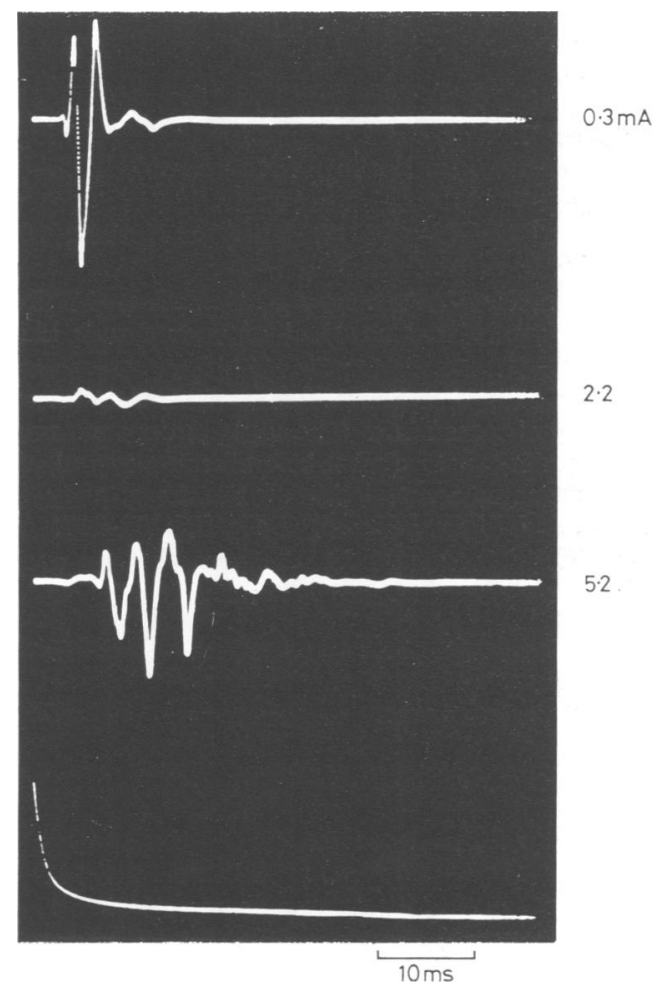

Fig 10 Anodal block of the lateral popliteal nerve using short exponentially-ending pulses and three symmetrically placed electrodes. EMG responses from the tibialis anterior. Baboon B104, three months after implantation.

\section{Peripheral nerve}

The experiment of fig 10 shows that the technique by which we produce anodal block in spinal roots works also in a peripheral nerve, the lateral popliteal. It works less well, the block being never quite complete. This may perhaps be related to the undulating course of fibre bundles in peripheral nerves.

\section{Discussion}

One function of the present paper is the scientific one of clarifying the conditions under which anodal block does and does not work. Its more 
important function is to establish a technique for blocking fibres through an implant. Such a technique will almost certainly have the clinical application to the treatment of detrusor-sphincter dyssynergia outlined in the Introduction. It may have other clinical uses, and will probably have applications in physiological research.

\section{References}

1 Pflüger E. Untersuchungen über die Physiologie des Electrotonus. Berlin: A Hirschwald, 1859.

2 Rushton WAH. The site of excitation in the nerve trunk of the frog. J Physiol (Lond) 1949; 109: 314-26.

3 Rushton WAH. A theory of the effects of fibre size in medullated nerve. J Physiol (Lond) 1951; 115:101-22.

4 Kuffler SW, Vaughan Williams EM. Small-nerve junctional potentials. The distribution of small motor nerves to frog skeletal muscle and the membrane characteristics of the fibres they innervate. J Physiol (Lond) 1953; 121:289-317.

5 Gotch F, Macdonald JS. Temperature and excitability. J Physiol (Lond) 1896; 20:247-97.
6 Burke W, Ginsborg BL. The electrical properties of the slow muscle fibre membrane. J Physiol (Lond) 1956; 132:586-98.

7 Accornero N, Bini G, Lenzi GL, Manfredi M. Selective activation of peripheral nerve fibre groups of different diameter by triangular shaped stimulus pulses. J Physiol (Lond) 1977; 273:539-60.

8 Brindley GS, Craggs MD. One-way stimulation of the large fibres of spinal roots through chronically implanted electrodes. J Physiol (Lond) 1978; 281: $12 \mathrm{P}$.

9 Brindley GS. An implant to empty the bladder or close the urethra. J of Neurol Neurosurg Psychiatry 1977 ; 40:358-69.

10 Thomas DG Smallwood R, Graham D. Urodydynamic observations following spinal trauma. Br J Urol 1975; 47:161-75.

11 Renshaw B. Influence of discharge of motoneurons. J of Neurophysiol 1941; 4:167-83.

12 Eccles JC, Fatt P, Koketsu K. Cholinergic and inhibitory synapses in a pathway from motoraxon collaterals to motoneurons. $J$ of Physiol (Lond) 1954; 216:524-62.

13 Van den Honert C, Mortimer JT. Generation of unidirectionally propagated action potentials in a peripheral nerve by brief stimuli. Science 1979; 206:1311-12. 


\section{Book reviews}

Neurotransmitters, Receptors and Drug Action Edited by Walter B Essman (pp 209; £17.95) Lancaster: MTP Press Ltd, 1980.

To compile a volume dealing with any aspect of neurotransmitter receptor function within the brain should deter most authors. The concepts governing this field change so rapidly that any book on the subject is by definition out of date before publication. Indeed, Neurotransmitters, Receptors and Drug Action has suffered this fate. References quoted seldom are later than 1978 and the ideas put forward, although then current, at times seem to be from the distant past. No blame for this can be attributed to either the editor or authors, rather the long delays suffered by many of us through the process of publication itself must be considered responsible. The volume also contains some strange bedfellows. Chapters deal with a variety of neurotransmittersacetylcholine, noradrenaline, dopamine, 5HT, GABA and histamine - but each is examined from the particular viewpoint of the individual authors such that the contents do not blend to produce a homogenous work. This might explain the need for the all embracing title used for the volume. Overall, therefore, this is not a book to be recommended for a current view of neurotransmitter receptor function within the brain. At almost $£ 18$ I would be surprised if many found it to be of use. Perhaps others contemplating similar volumes would be advised to wait until the instability of receptor theory lessens and the speed of publication improves.

$P$ JENNER

The Spinal Cord and Its Reaction to Traumatic Injury Edited by William F Windle (pp 368; SFr 95) New York: Marcel Dekker Inc, 1980.

This book has 16 authors, although it is not the result of a symposium. Chapter 1 is on history. Chapters 2 to 7 are on anatomy and embryology, chapters 8 to 12 on physiology, chiefly microphysiology of the cat, and chapters 13 to 18 on experimental pathology. Chapter 20 is on general pathology, and chapter 19 on early surgical interven- tion, as practised in the USA, in the management of spinal injuries.

Some chapters review the whole of a natural topic, but some are narrow accounts of the author's research and its immediate background. Human spinal tracts are presented in chapter 5 as if nothing new had been discovered about them since 1938. A whole chapter is devoted to the distribution of GABAergic neurones in the rat's spinal cord, but chemical transmitters other than GABA are neglected; noradrenaline is mentioned solely in a context of experimental pathology, and acetylcholine gets no mention at all. I enjoyed Chapter 4, which is an economically written 10-page summary of Dommisse's monograph on the blood supply of the cord, and chapter 17, in which Kao soberly and clearly describes his work on bridging the transected spinal cord with sciatic nerve graft. That such a graft gets invaded by CNS axons is well established and independently confirmed; what happens later remains obscure. This is a patchy book, good in parts, but not one that will greatly help the clinician who deals with spinal injuries.

GS BRINDLEY

Adaptation et restauration des fonctions nerveuses By Marc Jeannerod and Henri Hecaen (pp 323; unpriced) Villeurbanne: SIMEP, 1979

The best way to sum up this book would be to say that if it were in English it would be excellent. Alas, it is in French and I could not recommend it to anyone other than a fluent francophone.

The work itself is a comprehensive and scholarly review comprising 323 pages of closely typed text and listing over 1500 references, the most recent being dated 1978. The subject matter is in two parts. The first deals with the adaptability of the nervous system both in animals and humans. The chapter headings are historical review, sensory deprivation, peripheral motor system, deafferentation, vestibulo-ocular reflex, motor coordination and neuronal basis of learning. The second and larger part of the book deals with the recovery of function after a cerebral lesion in animals and man. The main headings are factors affecting outcome, restoration of visual function, recovery of motor func- tions, return of language and the anatomical and physiological basis of recovery.

ALAN WHITELEY

\section{Correction}

"A technique for anodally blocking large nerve fibres through chronically implanted electrodes" by GS Brindley and MD Craggs, Vol 43, pp 1083-90. Owing to a printers error the legend to fig 1 in this article was incorrect. The original legend read "Implanted electrodes: each implanted electrode-mount consists of a silicone-rubber body with two pairs of parallel channels closed at the top by means of silicone rubber flaps. Each channel of the rostral pair contains three symmetrically placed platinum electrodes $(a-b-c)$, and each channel of the caudal pair contains three asymmetrically placed electrodes (d-e-f). The outer electrodes of each set of three are usually connected together as a common anode. Contact with the electrodes is made through helically wound cables. The nearer channel is ready to receive a nerve whilst the other is shown occupied."

In addition, the lettering to the right of the first trace of figure $6 \mathrm{~A}$ should have read $0.1 \mathrm{~ms}$, not $0.1 \mathrm{~mA}$ as printed.

\section{Notices}

International Meeting of Neurological Sciences. This will be held in Bombay, India, 28-30 September 1981. Information may be obtained from Professor Manik Shahani, ECI Institute of Electrophysiology, Dr E Borges Marg, Parel, Bombay 400 012, India.

The Volvo Awards for low back pain research. The Volvo Company of Göteborg, Sweden is offering three prizes of US\$3000 each for papers submitted on: (1) Clinical studies, (2) Bioengineering studies, or (3) Studies in other basic science areas. Details are available from: Professor Alf Nachemson, Sahlgren Hospital, S-413 45, Göteborg, Sweden. 